\title{
Effectiveness and safety of rhIGF1 therapy in patients with or without Laron syndrome
}

\section{Peter Bang ${ }^{1}$, Joachim Woelfle², Valerie Perrot ${ }^{3}$, Caroline Sert ${ }^{3}$ and Michel Polak ${ }^{4}$ on behalf of the Eu-IGFD Registry Study Group}

${ }^{1}$ Division of Pediatrics, Department of Biomedical and Clinical Sciences, Faculty of Health Sciences, Linköping University, Linköping, Sweden, ${ }^{2}$ Children's Hospital, University of Erlangen, Erlangen, Germany, ${ }^{3}$ Ipsen Pharma, Boulogne-Billancourt, France, and ${ }^{4}$ Department of Paediatric Endocrinology, Gynaecology, and Diabetology, AP-HP, Necker-Enfants Malades University Hospital, IMAGINE Institute, University of Paris, Paris, France

Correspondence should be addressed to P Bang

Email

peter.bang@liu.se

\begin{abstract}
Objective: The European Increlex ${ }^{\circledR}$ Growth Forum Database Registry monitors the effectiveness and safety of recombinant human insulin-like growth factor-1 (rhIGF1; mecasermin, Increlex ${ }^{\circledR}$ ) therapy in patients with severe primary IGF1 deficiency (SPIGFD). We present data from patients with and without a reported genetic diagnosis of Laron syndrome (LS).

Design: Ongoing, open-label, observational registry (NCT00903110).

Methods: Children and adolescents receiving rhIGF1 therapy from 10 European countries were enrolled in 2008-2017 $(n=242)$. The treatment-naïve/prepubertal (NPP) cohort $(n=138)$ was divided into subgroups based on reported genetic diagnosis of LS $(n=21)$ or non-LS $(n=117)$. Multivariate analysis of the NPP-non-LS subgroup was conducted to identify factors predictive of growth response (first-year-height standard deviation score (SDS) gain $\geq 0.3$ ).

Assessments included change in height and weight over 5 years and adverse events (AEs).

Results: Height SDS gain from baseline was greater in the NPP-LS than the NPP-non-LS subgroup after 1 years' treatment $(P<0.05)$. In the NPP-non-LS subgroup, 56\% were responders; young age at baseline was a positive independent predictive factor $(P<0.001)$. NPP-non-LS-responders and the NPP-LS subgroup had a similar mean age (6.07 years vs 7.00 years) at baseline and height SDS gain in year 1 (0.64 vs 0.70), although NPP-non-LS-responders were taller $(P<0.001)$ at baseline. BMI SDS changes did not differ across subgroups. Treatment-emergent AEs were experienced by $65.3 \%$ of patients; hypoglycaemia was most common.

Conclusions: In most NPP children with SPIGFD, with or without LS, rhIGF1 therapy promotes linear growth. The safety profile was consistent with previous studies.
\end{abstract}

\section{Introduction}

Severe primary insulin-like growth factor deficiency (SPIGFD) is associated with postnatal growth failure (1). It is characterised by very low levels of insulin-like growth factor-1 (IGF1) and other growth hormone (GH)-regulated proteins, despite normal or elevated GH secretion $(2,3)$. In patients with Laron syndrome (LS), SPIGFD results from a mutation in the $\mathrm{GH}$ receptor gene, causing $\mathrm{GH}$ Printed in Great Britain insensitivity and severe-to-extreme short stature ( -4 to -10 height standard deviation score (SDS), increasing with age) $(2,3)$. In addition to proportionate short stature, phenotypic characteristics of LS include frontal bossing, hypoplastic midface, central obesity, small genitalia and delayed puberty $(4,5)$. Other genetic causes of SPIGFD, include STAT5b and acid-labile subunit (ALS) mutations 
$(6,7)$. However, most patients with severe growth stunting and biochemical characteristics of SPIGFD cannot be assigned a causative genetic diagnosis (8).

Recombinant human IGF1 (rhIGF1; mecasermin, Increlex ${ }^{\circledR}$; Ipsen Pharma, Boulogne-Billancourt, France) therapy stimulates linear growth in children with SPIGFD $(9,10)$ and improves adult height (11). In Europe, rhIGF1 therapy was approved for treating growth failure in children with SPIGFD in 2007 (12). The European Increlex ${ }^{\circledR}$ Growth Forum Database (Eu-IGFD) Registry was set up to collect long-term safety and effectiveness data from clinical practice (13).

In clinical trials, growth response to rhIGF1 therapy was shown to be dose-dependent (9). However, data collected from the Eu-IGFD Registry up to September 2013 demonstrated that rhIGF1 therapy effectiveness varied between subgroups (13). In patients who were treatmentnaïve/prepubertal, a younger age and lower baseline height SDS, characteristics of the LS subgroup included in the analysis, were predictors of a greater change in height SDS in year 1 of rhIGF1 therapy (13). Further analyses to determine and verify predictive response factors for patients with SPIGFD without LS would help to inform clinicians' treatment decisions.

The objective of the presented primary analyses was to evaluate the effectiveness and safety of up to 5 years of rhIGF1 therapy in children with SPIGFD, with or without a reported genetic diagnosis of LS. However, as most participants in the Eu-IGFD Registry do not present with LS, it would be beneficial to better characterise clinical characteristics of these patients. Therefore, the secondary analyses aimed to better describe baseline characteristics of patients without LS in whom rhIGF1 therapy was most effective, in order to support clinicians in their treatment decisions.

\section{Methods}

\section{Study design}

The Eu-IGFD Registry is an ongoing, open-label, observational study monitoring rhIGF1 therapy use in children with growth failure due to SPIGFD (NCT00903110). Enrolment criteria, study procedures and assessments have been reported previously (13). The EuIGFD Registry, started in 2008, includes children and adolescents with growth failure from 10 European countries, receiving rhIGF1 therapy, who provided informed consent or assent, as appropriate. Diagnosis of SPIGFD was judged by the reporting physician and was not possible to reassess for reasons previously detailed (13). Baseline was the visit closest to rhIGF1 therapy start. The cut-off date for analyses was 10 May 2017 (ENCEPP/ SDPP/7708).

The Eu-IGFD Registry is conducted in compliance with independent ethics committees/institutional review boards (except the UK, where the ethical review is not required for this registry type), informed consent regulations, the Declaration of Helsinki, International Conference on Harmonization, and Good Epidemiological Practice Guidelines.

\section{Patient subgroups}

Patients were divided into two cohorts (Fig. 1), that is, treatment-naïve and prepubertal (NPP), and not treatment-naïve and/or pubertal (non-NPP). Patients receiving recombinant human growth hormone (rhGH), rhIGF1 therapy and steroids were classed as non-NPP. Prepubertal was defined as Tanner stage 1 genital or

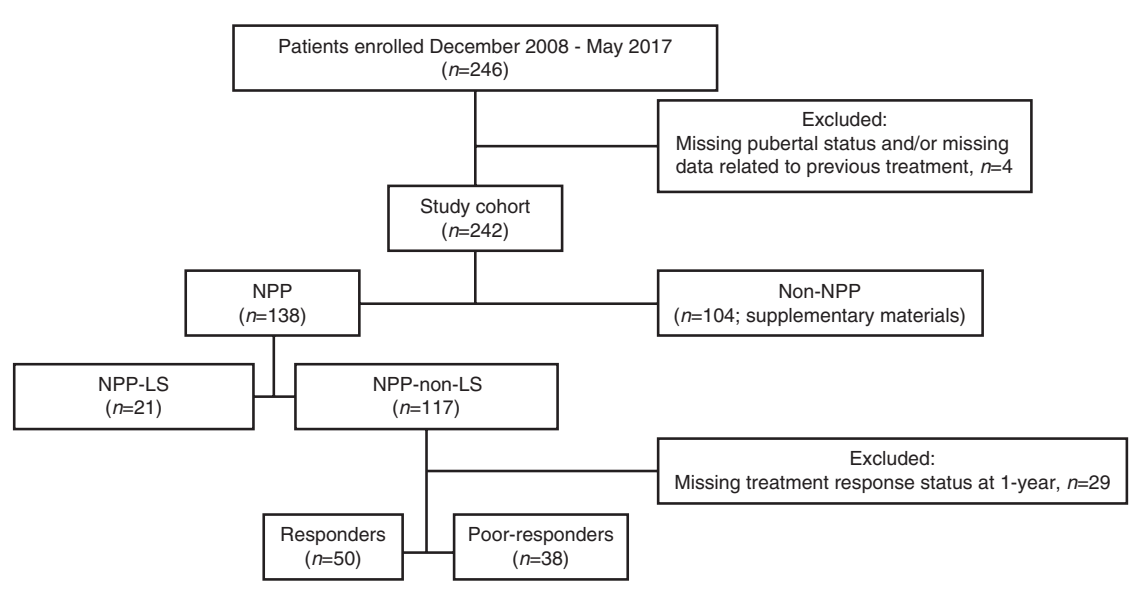

\section{Figure 1}

Study disposition and patient subgroup distribution. Responders were defined as patients with a change in height SDS in year 1 of $\geq 0.3$; poor-responders were defined as patients with a change in height SDS in year 1 of $<0.3$. In the registry and safety populations, three patients from the NPP-non-LS subgroup and one patient from the non-NPP cohort were excluded as no follow-up visits were performed. Non-NPP, not treatment naïve and/or pubertal; NPP, treatment-naïve and prepubertal; LS, Laron syndrome. 
breast development for boys and girls, respectively. Within each cohort, patients were split into two subgroups based on whether they had genetically verified LS (LS or non-LS). One patient reported with LS was diagnosed based on a typical phenotype, which was not genetically verified. For all other patients with LS, a GH receptor deletion or mutation was confirmed by genetic analysis.

\section{Safety analyses}

All adverse events (AEs), serious AEs, and treatmentemergent AEs (TEAEs) were analysed. Targeted TEAEs (13) were defined as AEs occurring while treatment was ongoing, which were either frequently or historically associated with rhIGF1 therapy (regardless of whether they were considered drug related).

\section{Statistical analysis}

The enrolled population (baseline characteristics data) comprised patients who had complete, clinical research associate-monitored baseline data (13); the registry population (effectiveness data) comprised patients who received $\geq 1$ dose of rhIGF1 therapy and completed $\geq 1$ follow-up visit; and the safety population (safety data) comprised patients who received $\geq 1$ dose of rhIGF1 therapy and who attended $\geq 1$ follow-up visit, or for whom there were post-study treatment safety data.

Calculations of weight, BMI, height SDS, and height velocity were performed as reported previously (13). Results from descriptive analyses are presented as mean (s.D. (95\% CI)) and/or median (first and third quartile: Q1; Q3). Categorical data were analysed using Chi-square or Fisher's exact test and quantitative data were analysed using ANOVA or Wilcoxon test. Statistical analyses were exploratory so no adjustment for multiplicity was performed.

\section{Univariate and multivariate analyses}

Logistic regression analysis was used to identify factors predictive of growth response at year 1 by comparing NPP-non-LS-responders with poor-responders (gain in height of $\geq 0.3$ SDS or $<0.3$ SDS after 1 year of treatment, respectively; Fig. 1). The cut-off was based on the previously reported mean change in height of the registry population at year 1 , and suggested cutoffs in patients with GH insensitivity $(13,14,15)$.
A univariate analysis used the following potential factors: sex, mid-parental adult height, birth height; and the following baseline parameters: age, height SDS, weight SDS, IGF1 level, IGF binding protein-3 and rhIGF1 therapy initial dose and dose during year 1 . Potentially important factors identified from the univariate analysis (significant at the 20\% level) were included in the final multivariate model.

\section{Results}

Overall, 246 patients enrolled in the Eu-IGFD Registry from December 2008 to May 2017, and 138 were included in the NPP cohort analyses; with LS $(n=21)$, or without LS $(n=117$; Fig. 1). The heterogeneity of the non-NPP cohort ( $n=104)$ did not allow for meaningful efficacy reporting. The non-NPP cohort baseline characteristics, puberty and previous treatment status, rhIGF1 therapy dosing and changes in height SDS, height velocity, BMI SDS and weight SDS are provided in Supplementary materials (Supplementary Tables 1, 2, 3, 4, 5 and 6, see section on supplementary materials given at the end of this article). All baseline and efficacy data reported below refer to the NPP cohort.

\section{Primary analyses: effectiveness of rhIGF1 therapy in NPP patients with or without LS}

Baseline characteristics of LS and non-LS subgroups are presented in Table 1 . The LS subgroup was significantly younger $(P=0.006)$, had a more severe short stature $(P<0.001)$, lower IGF1 levels $(P=0.007)$, and higher GH secretion $(P=0.014)$ compared with the non-LS subgroup at baseline. History of spontaneous hypoglycaemia before rhIGF1 therapy was more frequently observed in those with LS compared with those without LS: 4 (19\%) and 3 $(2.6 \%)$ patients, respectively $(P=0.011)$. The LS subgroup had a significantly higher rhIGF1 therapy starting dose compared with the non-LS subgroup (median (Q1; Q3): 40 (40; 40) vs 40 (20;40) $\mu \mathrm{g} / \mathrm{kg}$ BID; $P=0.013)$, but no significant difference in dose was observed from year 1 to year 5 after dose escalation. Duration of treatment was not significantly different between those with and without LS (median (Q1; Q3): 4.97 (2.09; 6.35) vs 3.90 $(1.98 ; 5.28)$ years).

While height SDS gain in the LS subgroup was observed during the first 2 years of treatment, the non-LS subgroup appeared to gain height SDS during subsequent 


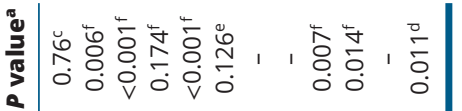

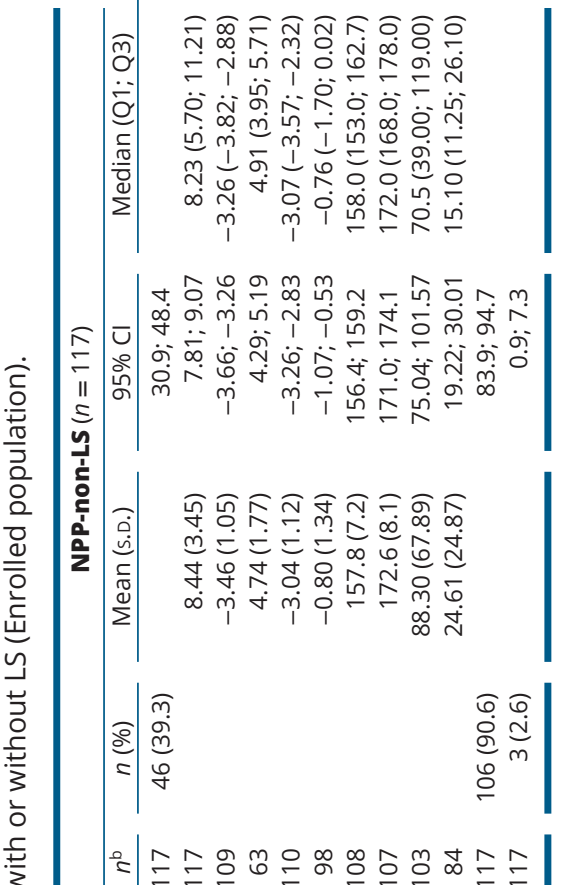

years (Fig. 2, Table 2 and Supplementary Table 4). Height SDS gain was significantly greater in the LS subgroup compared with the non-LS subgroup at year $1(P=0.019)$ and year $2(P=0.044)$. However, in years 3,4 and 5 , height SDS gain was not significantly different between the subgroups. There was also an apparent greater increase in BMI SDS and weight SDS from baseline in the LS subgroup vs the non-LS subgroup; however, weight SDS was only significantly greater in the LS subgroup at year 4 $(P=0.022)$ (Fig. 2 and Supplementary Tables 5,6$)$.

\section{Predictive factors of rhIGF1 therapy response in the NPP non-LS-subgroups}

To characterise factors predictive of growth response at year 1 in patients without LS, the NPP patients were divided into responders and poor-responders. Just over half of patients were responders (56.8\%; Fig. 1). The univariate analysis demonstrated that in NPP patients without LS, age, baseline IGF1, and mid-parental adult height were potentially important factors predictive of growth response at year 1 . However, the final multivariate analysis only identified a statistically significant correlation between the change in height SDS during year 1 and age at baseline (odds ratio: 0.75 (95\% CI 0.65; 0.87); $P<0.001$ ), with younger patients being better responders to rhIGF1 therapy than older patients. Responders were significantly younger than poor-responders (by approximately 4.5 years (median); $P<0.001$; Table 3 ); however, all other baseline characteristics, including height SDS, were similar between subgroups.

\section{Secondary analyses: NPP-non-LS-responders, poor-responders and NPP-LS subgroup comparison}

The similarities and differences between the two NPP subgroups that responded best to rhIGF1 therapy were analysed: patients with LS and responders without LS. At baseline, responders without LS and patients with LS were of similar age. However, patients with LS had a significantly lower height SDS, weight SDS, and serum IGF1 level, and higher peak stimulated GH levels, compared with responders without LS $(P<0.001, P<0.001, P=0.027$ and $P=0.025)$. Responders started on a slightly lower rhIGF1 therapy starting dose compared to patients with LS $(P=0.011)$ but with no difference observed from year 1 to year 5 . Responders were treated for a similar duration compared to patients with LS $(P=0.990)$.

During year 1 , responders without LS had a similar mean (S.D.) change in height SDS vs patients with LS (0.64 


\section{$\square$ Baseline $\square$ year $\square$ years $\square 3$ years $\square 4$ years $\square 5$ years}

A

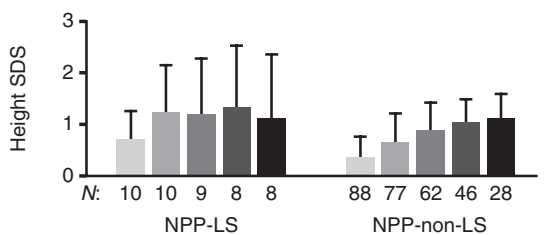

C

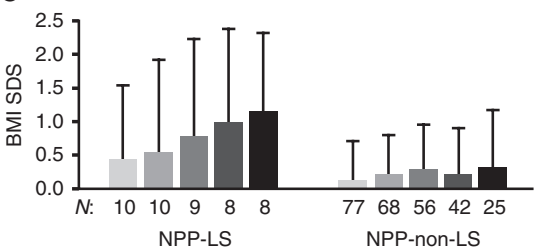

B 12

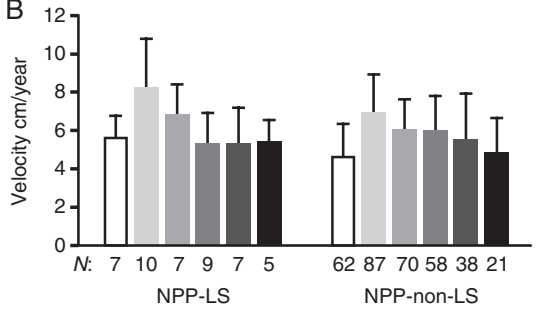

D

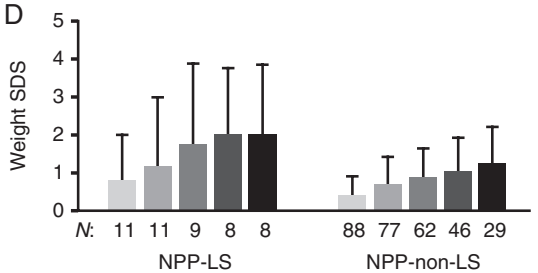

Figure 2

Effect of rhIGF1 therapy on height SDS (A), height velocity (B), BMI SDS (C), and weight SDS (D) in treatment-naïve/prepubertal (NPP) patients with or without LS (registry population). Data are mean (s.D.). $n$, number of patients with available data at each time point; LS, Laron syndrome; SDS, standard deviation score.
(0.26) vs $0.70(0.56) ; P=0.835)$. This was also reflected in the mean first year height velocities (Supplementary Table 4); mean height SDS gain was greatest in year 1 and continued to steadily increase in years 2-3 then remained stable in years 3-5 (Table 2). Other than BMI SDS at year $4(P=0.032)$, changes in BMI SDS and weight SDS from baseline were not significantly different between subgroups (Supplementary Tables 5 and 6).

Among NPP patients without LS, poor-responders and responders had similar rhIGF1 therapy doses from baseline to year 5; however, duration of treatment was significantly shorter in poor-responders (median: 3.47 vs 4.59 years; $P=0.028)$. There were no significant differences in BMI SDS changes at any time point between responders and poor-responders (Fig. 3). However, corresponding to the increase in height, responders had a significantly greater increase in weight SDS compared with poor-responders each year, except at year 4 (years 1-2: $P<0.001$; year 3 : $P<0.017$; year 5: $P=0.038$ )

\section{Safety profile}

An overview of TEAEs (including the non-NPP cohort; $n=242$ ) is presented in Table 4 . Overall, $65.3 \%$ of patients experienced a TEAE, 20.2\% experienced a serious TEAE and $5.4 \%$ had a TEAE that led to treatment withdrawal. The most frequently reported TEAEs were hypoglycaemia $(n=93)$, headache $(n=41)$, lipohypertrophy $(n=35)$ and middle ear infection $(n=26)$. The most common serious TEAEs were hypoglycaemia $(n=6(2.5 \%))$, followed by adenoidal and tonsillar hypertrophy (both: $n=4(1.7 \%)$ ). There were 3 benign and 1 malignant neoplasm TEAEs. The benign neoplasia events were mild in nature: dysplastic naevus occurred in 1 patient (0.4\%) (NPP-non-LS-poorresponder; no pre-existing conditions) and melanocytic naevus occurred in 2 patients (0.8\%) (NPP-non-LSresponder and NPP-non-LS-poor-responder; pre-existing asthma and no pre-existing conditions, respectively). The malignant event was fatal myelodysplastic syndrome which occurred in 1 patient (non-NPP-non-LS; multiple pre-existing conditions, including thrombocytopenia), as reported by Bang et al. (13). One other TEAE was fatal: a complication of a bone marrow transplant in a patient classified as NPP-responder without LS.

Within the treatment naïve/prepubertal cohort, there was an apparent higher frequency of targeted TEAEs in patients with LS (71.4\%) compared with those without LS (46.5\%; responder: $48.0 \%$, poor-responder: $36.8 \%$ ). The distribution of total and serious targeted TEAEs across the NPP cohort is presented in Fig. 4.

\section{Discussion}

These analyses demonstrate that rhIGF1 therapy promotes linear growth in children with SPIGFD but its effectiveness varies among subgroups of patients. As previously reported (16), NPP patients with LS responded significantly better to rhIGF1 therapy vs NPP patients without LS. However, the severity of GH insensitivity and genetic diagnosis confirming LS were not the only factors to determine a good response to rhIGF1 therapy. Among NPP patients without LS, a majority of younger patients with less severe signs of GH insensitivity responded similarly well to rhIGF1 therapy as the subgroup of NPP patients with LS. This finding was in accordance with our previous 
observation that young age is a positive predictor of a good height response in patients with SPIGFD, although this analysis included patients with LS (13).

\section{Primary analyses: effectiveness of rhIGF1 therapy in patients with or without LS}

The Eu-IGFD registry has a relatively large enrolment of patients with LS and, to our knowledge, there have been no other real-world studies that compare NPPl patients with LS vs those without LS. As expected, within the NPP cohort, the LS subgroup was significantly younger, had a more severe short stature, lower IGF1 levels and higher GH secretion at baseline compared with those without LS. Also in line with expectations, the LS subgroup responded significantly better to rhIGF1 therapy, in terms of height SDS gain, compared with patients without LS after 1-2 years of treatment. Conversely, in years 3-5, height SDS remained stable in the LS subgroup, whereas height SDS continued to increase in those without LS. However, this finding could be due to patients leaving the study, as patients without LS who ended treatment before year 3 are likely to have been those with the poorest response. Timing of puberty may also impact the interpretation of long-term growth response to rhIGF1 therapy, as patients with LS are reported to have delayed pubertal development (5).

Excessive weight gain has previously been reported in patients with LS and SPIGFD rhIGF1 therapy trials (5, 17), but this may have been due to encouraged snacking to prevent hypoglycaemia (12). Interestingly, a moderate increase in BMI ( 1.0 SDS) was observed over $4-5$ years of treatment in NPP patients with LS, which is less than reported by Backeljauw et al. (11), but greater than described in a case report of two slightly older patients (18) and greater than responders without LS in this analysis. Further research in this area would be of interest, to show whether the BMI trends observed here are significant in a larger patient population.

\section{Predictive factors of rhIGF1 therapy response in the NPP-non-LS subgroup}

We adopted a cut-off level for the response to treatment (gain in height SDS: 0.3) that could be considered unsatisfactory for most patients with an approved indication for rhGH treatment. As previously argued, rhIGF1 therapy in SPIGFD is unable to compensate for IGF1 independent actions of GH on growth (13). Furthermore, a similar cut-off was suggested for patients 


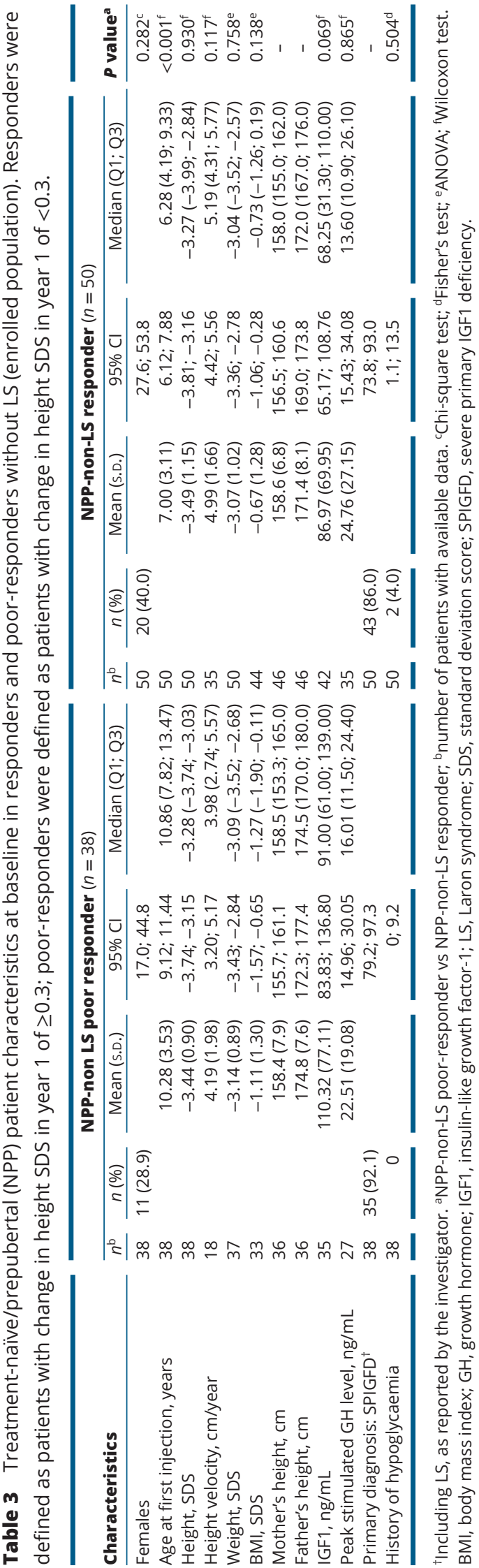

with a genetic defect in the IGF1 receptor treated with rhGH, a condition expected to have lower responsiveness than approved GH indications $(14,15)$.

Importantly, over half of NPP patients without LS were rhIGF1 therapy responders, with similar treatment response to those in a Polish study of patients without a LS phenotype $(n=27)$ (19). However, age at the start of treatment was the only baseline characteristic that predicted a better response to treatment in this population. Responders were approximately 4.5 years younger than poor-responders. Given that those untreated children with SPIGFD experience a decrease in height SDS as they age (20), these data suggest that rhIGF1 therapy initiation is delayed in children with less severe short stature. Furthermore, the limited gain in height SDS over 5 years in poor-responders underlines the importance of evaluating height response and TEAEs after 1 year and considering whether to stop or continue treatment.

\section{Secondary analysis: NPP-non-LS-responders and NPP-LS subgroup comparison}

Interestingly, patients in the NPP subgroup with LS and responders without LS started rhIGF1 therapy at a similar age, despite patients in the LS subgroup having a more severe short stature, lower IGF1 levels and higher GH secretion at baseline. These data suggest that the severity of short stature and clear biochemical abnormalities may not always lead to early treatment initiation in clinical practice. Notably, our data show that responders in the subgroup of NPP patients without LS had a similar initial and long-term mean gain in height SDS as those with LS . Thus, in SPIGFD, a genetic diagnosis is not necessarily required for a clinically significant height response. However, the LS subgroup remained shorter in stature than responders without LS. In this respect, the height response to rhGH therapy in patients with severe GH deficiency is more marked $(21,22)$.

\section{Non-NPP subgroups}

The heterogeneity of the non-NPP cohort does not allow for meaningful reporting; therefore, we refrain from interpreting the baseline and effectiveness of rhIGF1 therapy data in these patients.

\section{Safety profile}

Overall, safety data were consistent with the known profile of rhIGF1 therapy $(9,13)$. Hypoglycaemia was 
a Baseline $\quad 1$ year $\quad 2$ years $\square 3$ years $\square 4$ years $\square 5$ years

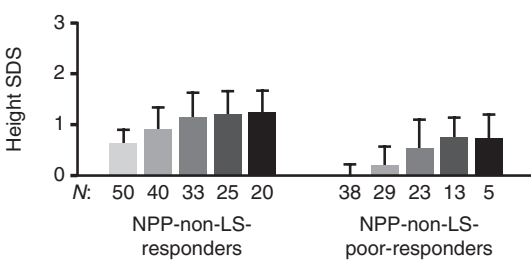

C

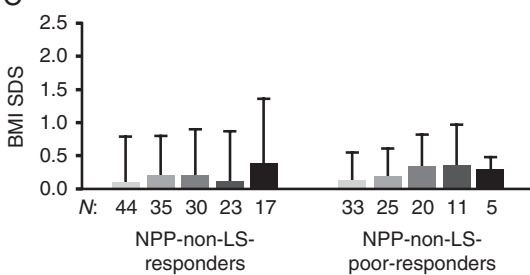

B
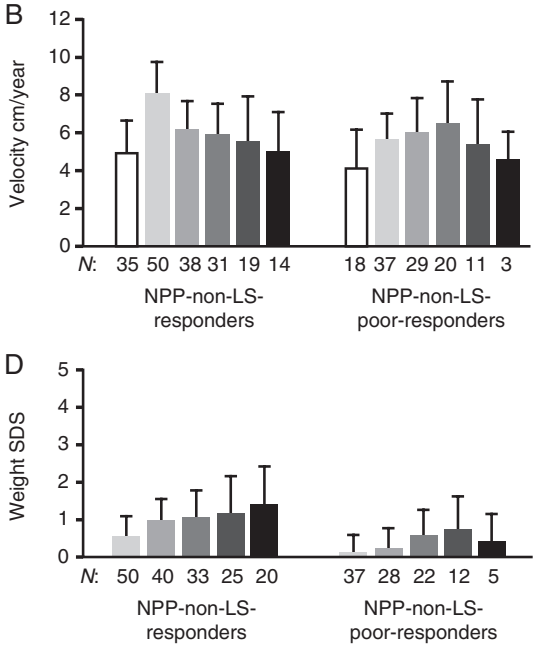

\section{Figure 3}

Effect of rhIGF1 therapy on height SDS (A), height velocity (B), BMI SDS (C), and weight SDS (D) in treatment-naïve/prepubertal (NPP) responders and poor-responders without LS (registry population). Data are mean (s.D.). Responders were defined as patients with a change in height SDS in year 1 of $\geq 0.3$. Poor-responders were defined as patients with a change in height SDS in year 1 of $<0.3$. $n$, number of patients with available data at each time point. LS, Laron syndrome; non-NPP, not treatment naïve and/or pubertal; SDS, standard deviation score. the most frequent TEAE and serious TEAE, in agreement with previous reports $(9,13)$, although hypoglycaemia is known to occur spontaneously in patients with LS (11). Targeted TEAEs were reported in a higher proportion of patients with than without LS.

Given that IGF1 has mitogenic and anti-apoptotic effects (23), rhIGF1 therapy may stimulate the growth of benign and malignant tumours that pre-exist or develop in patients with SPIGFD (12). Furthermore, IGF1 deficiency in untreated LS is thought to decrease the incidence of cancer (5). There have been post-marketing reports of both benign and malignant neoplasms in children and adolescents who have received rhIGF1 therapy; these cases represented a variety of different and rare malignancies (12). Although available data do not allow calculations of relative risk, the current analyses include four benign and one malignant neoplasm TEAEs. In those who receive rhIGF1 therapy for unapproved uses or at above the recommended doses, risk of neoplasia may be higher. Clinicians should be vigilant for potential malignancy symptoms and if neoplasia develops, rhIGF1 therapy should be discontinued definitely and appropriate expert medical care sought. However, the data in this study do not raise any new safety concerns.

\section{Limitations}

Given the observational nature of the Eu-IGFD Registry, inherent limitations exist as reported earlier (13). The disparity in age between subgroups at baseline could be considered a limitation; however, it supported our finding that age can be used to predict response to treatment. As previously reported, due to the number of different IGF1 assays used by the centres, it is not possible to determine how many patients fulfil the European diagnostic criteria of SPIGFD (IGF1 <2.5th percentile) (13). Measurements of IGF1 levels at baseline may have been confounded by the use of local rather than central laboratory analysis, as results obtained from different assays can vary considerably (24). Furthermore, IGF1 SDS was not available, therefore age could have impacted analyses of IGF1 levels. As data were collected from sites across 10 European countries, differences in diagnostic and treatment practices and in reporting standards are expected. International collaboration is needed to ensure a consistent approach to the identification and management of rare growth diseases such as SPIGFD (25). This analysis is limited by the small patient cohort, expected with a rare disease, therefore the statistical analysis should be interpreted with caution. Despite these limitations, the Eu-IGFD Registry provides valuable insights into the real-world effectiveness of rhIGF1 therapy in subgroups of clinical interest.

Table 4 Overall summary of treatment-emergent adverse events (safety population; $n=242$ ).

\begin{tabular}{l} 
Classification \\
\hline Patients with $\geq 1$ TEAE \\
Patients with $\geq 1$ targeted TEAE \\
Patients with $\geq 1$ serious TEAE \\
Patients with $\geq 1$ serious related TEAE \\
Patients with $\geq 1$ serious targeted TEAE \\
Patients with $\geq 1$ TEAE leading to \\
treatment withdrawal \\
Patients with a fatal TEAE
\end{tabular}

\begin{tabular}{c}
\hline $\boldsymbol{n}(\%)$ \\
\hline $158(65.3)$ \\
$119(49.2)$ \\
$49(20.2)$ \\
$26(10.7)$ \\
$15(6.2)$ \\
$13(5.4)$ \\
$2(0.8)$
\end{tabular}

TEAE, treatment-emergent adverse event. 
A

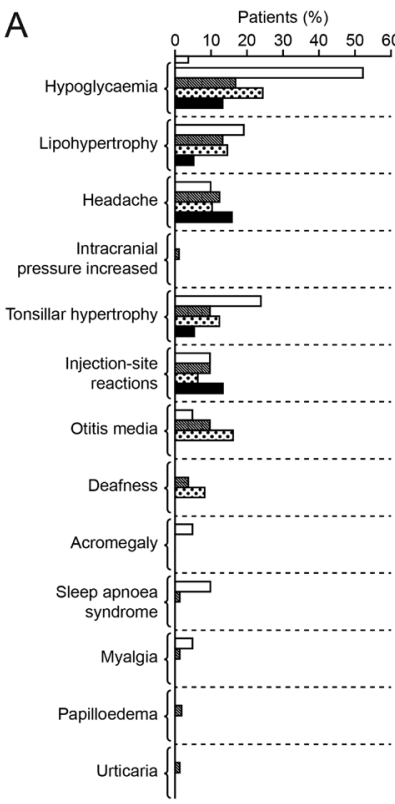

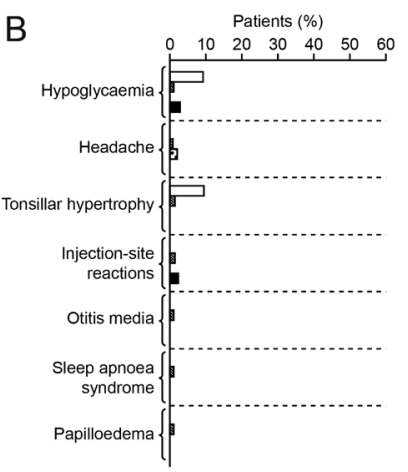

$\square$ NPP-LS ( $n=21)$ NPP-non-LS ( $n=114)$ Q 2 NPP-non-LS-responder $(n=50)$ - NPP-non-LS-poor-responder $(n=38)$

\section{Figure 4}

All targeted TEAEs for treatment-naïve/prepubertal (NPP) patient subgroups (A) total, (B) serious (safety population). All reported targeted TEAEs and serious targeted TEAEs are shown. Targeted TEAEs were: headache, chronic middle ear infection, papilledema, hypoglycaemia, acromegalic facial changes, oedema, gynaecomastia, hearing loss, intracranial hypertension, lipohypertrophy at injection sites, myalgia, sleep apnoea, tonsillar hypertrophy and cardiomegaly. Hearing impairment was coded as deafness. Responders were defined as patients with a change in height SDS in year 1 of $\geq 0.3$. Poor-responders were defined as patients with a change in height SDS in year 1 of $<0.3$. LS, Laron syndrome; TEAE, treatment-emergent adverse event.

\section{Conclusion}

Our analyses demonstrate that rhIGF1 therapy effectively promoted linear growth in the majority of NPP children with SPIGFD. Furthermore, young age at treatment initiation was a positive predictive factor of treatment response in NPP patients without LS, underlining the importance of starting treatment early. Moreover, NPP patients with LS and responders without LS had a similar initial and long-term clinically significant mean gain in height SDS, demonstrating a genetic diagnosis confirming LS was not necessarily a prerequisite for the response. The most common TEAE was hypoglycaemia, reported at a rate of 0.1 events and 0.01 serious events per treatment years. The data in this study do not raise any new safety concerns; however, clinicians and patients should remain

vigilant for benign or malignant neoplasia development and, if detected, discontinue treatment immediately and seek appropriate expert medical care.

\section{Supplementary materials}

This is linked to the online version of the paper at https://doi.org/10.1530/ EJE-20-0325.

\section{Declaration of interest}

P B: advisory board/board of directors fees from Ipsen, Lilly; and consulting fees from Ipsen, Sandoz, Pfizer, Lilly, Versatis. J W: advisory board/board of directors fees from Ipsen, Novo Nordisk; corporate-sponsored research fees from Pfizer, Ipsen; and speaker fees from Merck-Serono, Hexal, Pfizer, Novo Nordisk. V P and C S are employees of Ipsen. M P: advisory board/ board of directors fees from Ipsen (Increlex registry), Novo Nordisk (Global Norditropin Advisory board), Pfizer, France; corporate-sponsored research fees from Ipsen, Novo Nordisk, Pfizer, Sandoz, Merck, Sanofi; and speaker fees from Novo Nordisk, Ipsen.

\section{Funding}

This study is supported by Ipsen.

\section{Medical writing support}

The authors thank Cara Valvona, PhD, of Watermeadow Medical, an Ashfield Company, for providing medical writing support, which was sponsored by Ipsen in accordance with Good Publication Practice guidelines.

\section{Data sharing statement}

Where patient data can be anonymised, Ipsen will share all individual participant data that underlie the results reported in this article with qualified researchers who provide a valid research question. Study documents, such as the study protocol and clinical study report, are not always available. Proposals should be submitted to DataSharing@Ipsen. com and will be assessed by a scientific review board. Data are available beginning 6 months and ending 5 years after publication; after this time, only raw data may be available.

\section{Author contribution statement}

$\mathrm{PB}, J \mathrm{~W}, \mathrm{VP}, \mathrm{C}$ S and M P were involved in substantial contributions to study conception and design, acquisition and interpretation of data, drafting the publication and revising it critically for important intellectual content and final approval of the publication.

\section{Acknowledgements}

The authors thank all patients involved in the study, as well as their caregivers, care team, investigators and research staff in participating institutions. The authors also thank Haris Shaikh, Senior Safety Physician, Ipsen, for his review of the safety data and contribution to the manuscript.

\section{References}

1 Cohen J, Blethen S, Kuntze J, Smith SL, Lomax KG \& Mathew PM. Managing the child with severe primary insulin-like growth factor-1 
deficiency (IGFD): IGFD diagnosis and management. Drugs in $R \& D$ 201414 25-29. (https://doi.org/10.1007/s40268-014-0039-7)

2 Laron Z. Laron syndrome (primary growth hormone resistance or insensitivity): the personal experience 1958-2003. Journal of Clinical Endocrinology and Metabolism 200489 1031-1044. (https://doi. org/10.1210/jc.2003-031033)

3 Fintini D, Brufani C \& Cappa M. Profile of mecasermin for the long-term treatment of growth failure in children and adolescents with severe primary IGF-1 deficiency. Therapeutics and Clinical Risk Management 20095 553-559. (https://doi.org/10.2147/tcrm.s6178)

4 Laron Z, Pertzelan A \& Mannheimer S. Genetic pituitary dwarfism with high serum concentation of growth hormone - a new inborn error of metabolism? Israel Journal of Medical Sciences 19662 $152-155$.

5 Laron Z \& Kauli R. Fifty seven years of follow-up of the Israeli cohort of Laron syndrome patients-from discovery to treatment. Growth Hormone and IGF Research 201628 53-56. (https://doi.org/10.1016/j. ghir.2015.08.004)

6 Hwa V. STAT5B deficiency: impacts on human growth and immunity. Growth Hormone and IGF Research 201628 16-20.

7 Hwa V, Haeusler G, Pratt KL, Little BM, Frisch H, Koller D \& Rosenfeld RG. Total absence of functional acid labile subunit, resulting in severe insulin-like growth factor deficiency and moderate growth failure. Journal of Clinical Endocrinology and Metabolism 2006 91 1826-1831. (https://doi.org/10.1210/jc.2005-2842)

8 Grosse G, Hilger A, Ludwig M, Reutter H, Lorenzen F, Even G, Holterhus PM, Woelfle J \& German GHI Study Group. Targeted resequencing of putative growth-related genes using whole exome sequencing in patients with severe primary IGF-I deficiency. Hormone Research in Paediatrics 201788 408-417. (https://doi. org/10.1159/000480505)

9 Chernausek SD, Backeljauw PF, Frane J, Kuntze J, Underwood LE \& GH Insensitivity Syndrome Collaborative Group. Long-term treatment with recombinant insulin-like growth factor (IGF)-I in children with severe IGF-I deficiency due to growth hormone insensitivity. Journal of Clinical Endocrinology and Metabolism 200792 902-910. (https://doi.org/10.1210/jc.2006-1610)

10 Ranke MB, Savage MO, Chatelain PG, Preece MA, Rosenfeld RG, Blum WF \& Wilton P. Insulin-like growth factor I improves height in growth hormone insensitivity: two years' results. Hormone Research 199544 253-264. (https://doi.org/10.1159/000184637)

11 Backeljauw PF, Kuntze J, Frane J, Calikoglu AS \& Chernausek SD. Adult and near-adult height in patients with severe insulin-like growth factor-I deficiency after long-term therapy with recombinant human insulin-like growth factor-I. Hormone Research in Paediatrics 201380 47-56. (https://doi.org/10.1159/000351958)

12 EMA. Increlex - summary of product characteristics, 2020. (available from: https://www.ema.europa.eu/en/documents/productinformation/increlex-epar-product-information_en.pdf)

13 Bang P, Polak M, Woelfle J, Houchard A \& EU IGFD Registry Study Group. Effectiveness and safety of rhIGF-1 therapy in children: the European Increlex(R) Growth Forum database experience. Hormone Research in Paediatrics 201583 345-357. (https://doi. org/10.1159/000371798)

14 Walenkamp MJE, Robers JML, Wit JM, Zandwijken GRJ, van Duyvenvoorde HA, Oostdijk W, Hokken-Koelega ACS, Kant SG \& Losekoot M. Phenotypic features and response to GH treatment of patients With a molecular defect of the IGF-1 receptor. Journal of Clinical Endocrinology and Metabolism 2019104 3157-3171. (https:// doi.org/10.1210/jc.2018-02065)

15 Gopel E, Rockstroh D, Pfaffle H, Schlicke M, Pozza SB, GannageYared MH, Gucev Z, Mohn A, Harmel EM, Volkmann J et al. A comprehensive cohort analysis comparing growth and GH therapy response in IGF1R mutation carriers and SGA children. Journal of Clinical Endocrinology and Metabolism 2020105 dgz165. (https://doi. org/10.1210/clinem/dgz165)

16 Bang P, Polak M, Woelfle J, Houchard A \& Sert C. Safety and effectiveness of Increlex® therapy in children with Laron syndrome and enrolled in the European Increlex ${ }^{\circledR}$ Growth Forum Database (EU-IGFD) in Europe. Hormone Research in Paediatrics 201584 (Supplement 1) 43 abst FC7.1.

17 Laron Z. Emerging treatment options for patients with Laron syndrome. Expert Opinion on Orphan Drugs 20142 681-694. (https:// doi.org/10.1517/21678707.2014.912581)

18 Ekstrom K, Carlsson-Skwirut C, Ritzen EM \& Bang P. Insulin-like growth factor-I and insulin-like growth factor binding protein-3 cotreatment versus insulin-like growth factor-I alone in two brothers with growth hormone insensitivity syndrome: effects on insulin sensitivity, body composition and linear growth. Hormone Research in Paediatrics 201176 355-366. (https://doi. org/10.1159/000330410)

19 Petriczko E, Jackowski T, Horodnicka-Jozwa A, Wikiera B, Noczynska A, Korpal-Szczyrska M, Birkholz-Walerzak D, MaleckaTendera E, Kalina-Fraska B, Kalina M et al. Treatment of severe primary IGF-1 deficiency using rhIGF-1 preparation - first three years of Polish experience. Endokrynologia Polska 201970 20-27. (https:// doi.org/10.5603/EP.a2018.0074)

20 Laron Z. The essential role of IGF-I: lessons from the long-term study and treatment of children and adults with Laron syndrome. Journal of Clinical Endocrinology and Metabolism 199984 4397-4404. (https:// doi.org/10.1210/jcem.84.12.6255)

21 Bang P, Bjerknes R, Dahlgren J, Dunkel L, Gustafsson J, Juul A, Kristrom B, Tapanainen P \& Aberg V. A comparison of different definitions of growth response in short prepubertal children treated with growth hormone. Hormone Research in Paediatrics 201175 335-345. (https://doi.org/10.1159/000322878)

22 Bang P, Ahmed SF, Argente J, Backeljauw P, Bettendorf M, Bona G, Coutant R, Rosenfeld RG, Walenkamp MJ \& Savage MO. Identification and management of poor response to growthpromoting therapy in children with short stature. Clinical Endocrinology 201277 169-181. (https://doi.org/10.1111/j.13652265.2012.04420.x)

23 Brahmkhatri VP, Prasanna C \& Atreya HS. Insulin-like growth factor system in cancer: novel targeted therapies. BioMed Research International 20152015 538019. (https://doi. org/10.1155/2015/538019)

24 Frystyk J, Freda P \& Clemmons DR. The current status of IGF-I assays - a 2009 update. Growth Hormone and IGF Research 201020 8-18. (https://doi.org/10.1016/j.ghir.2009.09.004)

25 Bang P, Polak M, Woelfle J, Perrot V \& Sert C. The European Increlex ${ }^{\circledR}$ Growth Forum Database (EU-IGFD) registry: do treatment practices differ between European countries? Hormone Research in Paediatrics 201991 (Supplement 1) 233 (Abstract: P1-214). (https://doi.org/10.1159/000501868)
Received 17 April 2020

Revised version received 2 October 2020

Accepted 17 November 2020 